ISAHP 2005, Honolulu, Hawaii, July 8-10, 2003

\title{
A RESTAURANT PLANNING MODEL BASED ON FUZZY-AHP METHOD
}

\author{
Heung Suk Hwang ${ }^{1}$ \\ 1: Department of Business Administration, Kainan University, Taiwan \\ No. 1 Kainan Rd., Lu-jhu, Taoyuan, Taiwan 338, \\ e-mail : hshwang@mail.knu.edu.tw \\ Wen-Hwa Ko ${ }^{2}$ \\ 2: Department of Business Tourism and Hospitality Management, Kainan University, Taiwan \\ No. 1 Kainan Rd., Lu-jhu, Taoyuan, Taiwan 338, \\ e-mail : kowh@mail.knu.edu.tw
}

Keywords: Restaurant Location Planning, AHP, Stochastic Set-covering Problem

Summary: Recently, a new method of optimizing the restaurant planning for the decision of restaurant types, the locations and food service system. The multi-criteria decision analysis is one of the evident areas of important points in integrated planning of the restaurant service system.

This research is concerned with the development of a 3-step restaurant planning based on service level, multi-criteria decision analysis, and stochastic set-covering method. Following three researches are included; 1) optimal decision of restaurant types using analytic hierarchy process and fuzzy-AHP as a multi-criteria decision analysis method, 2) determine the optimal number of restaurant and those locations with minimum customers travel distance using set-covering problem, and 3) these procedures are shown on visual outputs by a computer program. The computer program is developed and demonstrated the computational results for school food service facility of Taoyuan area in Taiwan. It is known that the proposed method is very effective on a set of test problems.

\section{Introduction}

Restaurant facility planning is an import analysis leading to the success of restaurant management because it can determine the type and required number of restaurant with optimal locations. There are many studies performed on facility planning and location problems such as; Aly and White (1998), Appl and Rickleres (1987), Francis and White (1974), and Tomkins et al. (1996). However, the most of these researches are concerned with material handling systems. Although there are some of researches on foodservice operations' efficiency by focusing on variables affecting productivity such as; Brown and Hoover (1990), Clark (1997), Lieux and Winkler (1989), Manning and Lieux (1991), Manning and Lieux (1995), Mayo and Olsen (1984). These studies failed to provide a basis on which the comprehensive performance criteria in terms of integrated decision, goal achievement and identify relative weights of decision criteria are determined. Most of these models and researches have been developed for the design of facilities and its location problems. However these techniques provide invaluable information for integrated a decision system for restaurant facility planning, on the other hand this research is concerned with optimizing the location and service level for restaurant facilities planning based on analytic approach considering the customers required service level, optimal number and the possible locations of restaurant facilities. We develop a three-step approach using optimizing methods as explained following. In step 1, we decided the restaurant alternative such as the type and service level of the restaurant for the regional customers.

1. Corresponding Author 
In this step, we decide the type of facility alternative, possible locations, service levels considering all the facility characteristics, and we developed a 3-step solution builder and apply it to implement the facility alternative such as; 1) brainstorming model to generate the restaurant alternative, 2) multi-criteria decision analysis model to evaluate the individual alternatives using AHP (analytic hierarchy process) and fuzzy set ranking methodologies to overcome the special decision problems of restaurant, and 3) sector clustering model to cluster the customers for every restaurant optimizing total regional performance. In step 2, we determine the optimal number of restaurant facility needed to meet the customer's service level. We use a stochastic network simulation and 0-1 programming, and in step 3, we decide the groups of customers using sector clustering algorithm for each restaurant facility so as to guarantee to meet the required service level (Potvnt, 1996). Finally, we develop the computer programs for this procedure. Figure 1 shows the three-step approach of the decision support system for restaurant planning.

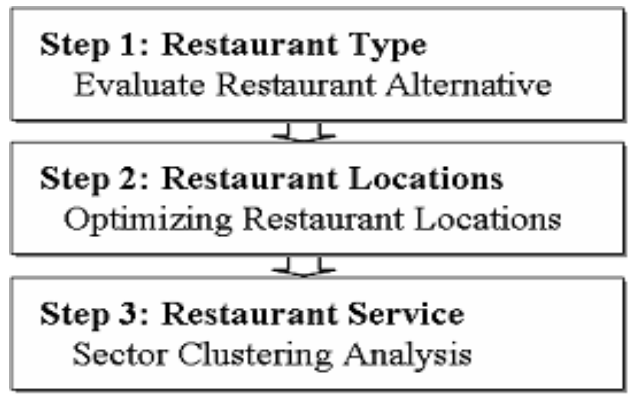

Figure 1. Three-step approach of restaurant planning

\section{Restaurant Type Evaluation Using Fuzzy-AHP}

\subsection{Web-Based Solution Builder}

We develop three steps decision solution builder to decide the types of restaurant. In the first step, we develop a brainstorming method based on an internet/intranet to create the ideas to drive out alternatives based on the group analysts, and in the second step, we evaluate the decision alternatives that derived out in the step 1 using the AHP method and determined the preferred alternative. In the last step, we integrate the results of individual evaluations into one ranked order. We developed two heuristic methods based on majority rule method for this last step. Figure 2 shows the schematic structure of three-step approach.

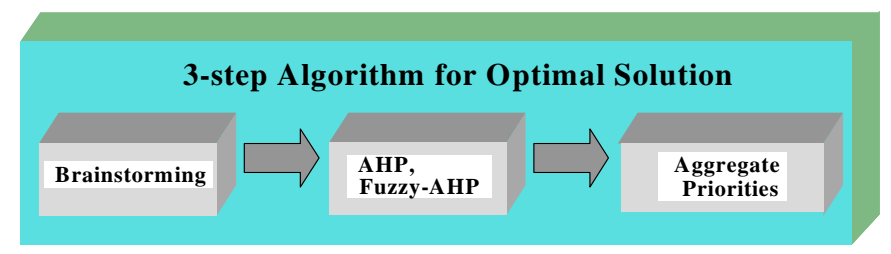

Figure 2. Structure of 3-step solution builder for decision support system

1) AHP and fuzzy-AHP method: In this study we used AHP and fuzzy-AHP method to evaluate individual restaurant alternatives. The fuzzy priority is computed and compared with the rank order of the other methods. The fundamental concept of fuzzy set priority relation, $\mathrm{R}$, is derived from the result obtained by Shannon (1986) method. From the Shannon's summed frequency matrix for complementary cells, $A_{i j}$ and $A_{j i}$, an additional fuzzy set matrix is made by considering $A_{i j}=1-A_{j i}$ for all cells.

To obtain fuzzy preferences, the following five steps are considered:

Step 1: Find the summed frequency matrix (using Shannon method)

Step 2: Find the fuzzy set matrix $\mathrm{R}$ which is the

Summed frequency matrix divided by the total number of evaluators 
Step 3: Find the difference matrix

$$
\begin{aligned}
& R-R^{T}=\mathrm{U}(\mathrm{A}, \mathrm{B})-\mathrm{U}(\mathrm{B}, \mathrm{A}) \text {, if } \mathrm{U}(\mathrm{A}, \mathrm{B})>\mathrm{U}(\mathrm{B}, \mathrm{A}), \\
& =0, \quad \text { otherwise }
\end{aligned}
$$

where, for $U(A, B)$ quantifies, $A$ is preferable to $B$.

Step 4: Determine the portion of each project that is not dominated as follows:

$$
A_{\text {ColA }}^{N D}=1-\max \left(X_{1 . \text { ColA }}, X_{2 . \text { ColA }}, \ldots, X_{n . \text { ColA }}\right)
$$

Step 5: The priority of the fuzzy set is then the rank order of XND values with a decreasing order. An example is shown as follows:

$$
\begin{aligned}
& R=\left[\begin{array}{llll}
0.0 & 0.8 & 0.6 & 0.6 \\
0.2 & 0.0 & 0.0 & 0.4 \\
0.4 & 0.1 & 0.0 & 0.4 \\
0.4 & 0.6 & 0.6 & 0.0
\end{array}\right] \quad R^{T}=\left[\begin{array}{llll}
0.0 & 0.2 & 0.4 & 0.4 \\
0.8 & 0.0 & 0.1 & 0.6 \\
0.6 & 0.0 & 0.0 & 0.6 \\
0.6 & 0.4 & 0.4 & 0.0
\end{array}\right] \\
& R-R^{T}=\left[\begin{array}{llll}
0.0 & 0.6 & 0.2 & 0.2 \\
0.0 & 0.0 & 0.0 & 0.0 \\
0.0 & 0.1 & 0.0 & 0.0 \\
0.0 & 0.2 & 0.2 & 0.0
\end{array}\right] \\
& X_{A}^{N D}=1-\operatorname{Max}(0.0)=1-0.0=1.0, \quad X_{B}^{N D}=1-\operatorname{Max}(1.0)=1-0.6=0.4 \\
& X_{C}^{N D}=1-\operatorname{Max}(0.2)=1-0.2=0.8, \quad X_{D}^{N D}=1-\operatorname{Max}(0.2)=1-0.2=0.8
\end{aligned}
$$

Thus, the fuzzy set priority score of this example is given by $1.0>0.8>0.8>0.4$ and the alternative priority is given by $\mathrm{A}>\mathrm{C}>\mathrm{D}>\mathrm{B}$.

2) Integration of Individual Evaluation: For the integration of the results of individual evaluations and prioritized sets, we use a heuristic method which is a kind of majority-rule and fuzzy set priority methods. This method is compared to other methods to determine the most preferred one for the decision support system purpose.

- Heuristic Model: In this method, the preference score is given by the sum of the marks received from the evaluators, where for $\mathrm{m}$ alternatives, the marks are given, in decreasing order preference, (m-1), (m$2), \ldots, 0$. The ranking is based on the scores of each alternative. In this case, the highest score is the first priority. For example of the heuristic method, a sample results of a problem with $\mathrm{N}=5$ evaluators and $\mathrm{M}$ $=3$ alternatives are given as Table 1 :

Table 1. Example result of heuristic method

\begin{tabular}{|c|c|c|c|}
\hline Alt. & Preference matrix & Raw sum & Weighed value \\
\hline School A & 0.01 .01 .0 & 2.0 & 0.133 \\
School B & 4.00 .02 .0 & 6.0 & 0.400 \\
School C & 4.03 .00 .0 & 7.0 & 0.467 \\
\hline Heuristic Method & C > B > A & & \\
Rank Order & & \\
\hline
\end{tabular}

Evaluator 1: $\mathrm{B}>\mathrm{A}>\mathrm{C}, \quad$ Evaluator 2: $\mathrm{B}>\mathrm{C}>\mathrm{A}, \quad$ Evaluator 3: $\mathrm{C}>\mathrm{A}>\mathrm{B}$, Evaluator 4: $\mathrm{C}>\mathrm{B}>\mathrm{A}, \quad$ Evaluator 5: $\mathrm{C}>\mathrm{B}>\mathrm{A}$

We developed the computer program and applied to a set of example problems of multi-structured restaurant planning problems.

\subsection{Sample Problem of Restaurant Alternative Evaluation}

For a sample problem of restaurant alternative evaluation using three step decision models, a new restaurant for school food service problem is considered. We used the model according to following three steps where we have to decide the restaurant type and evaluation factors such as; 1 ) In step 1, to decide the evaluation factors and restaurants alternatives using brainstorming process and we converted this result into AHP-evaluation structure automatically by program, 2) in step 2, using a heuristic and fuzzyAHP method, we find the eigen value by pair-wise comparison matrix. This evaluation is done by 4 
evaluation members and 3) in step 3, the results of individual evaluators are integrated by heuristic and fuzzy set priority methods. Following tables and figures summarized the sample outputs of this problem.

1) Brainstorming Module: In step 1, by brainstorming method we found the restaurant alternative and evaluation factors as following;

- Restaurant alternative (4 alternatives); full outsourcing type, partial owner and partial outsourcing type, short term contract type, and full own and operating type.

- Evaluation factors (3 factors); cost, quality, and food flexibility.

Figure 3 and 4 show the brainstorming output and converted into AHP structure respectively.

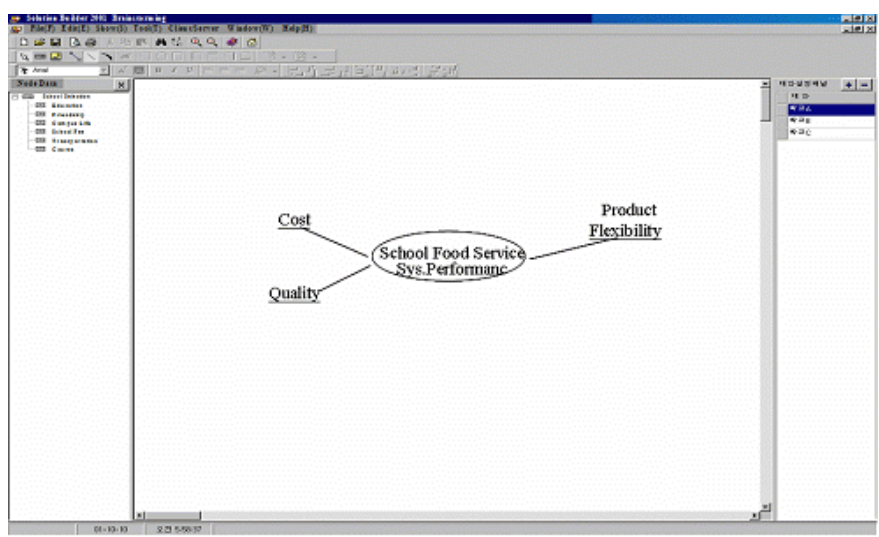

Figure 3. Sample output of brainstorming (Evaluation factors)

\section{2) Evaluation of Alternative: Step 2}

Figure 4 and 5 show the sample output of this example problem.

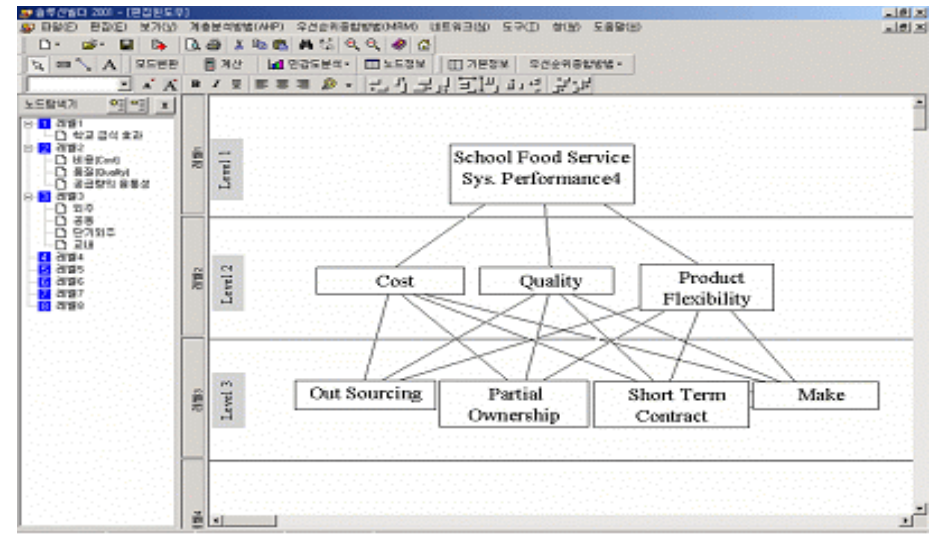

Figure 4. Sample output of AHP structure diagram 


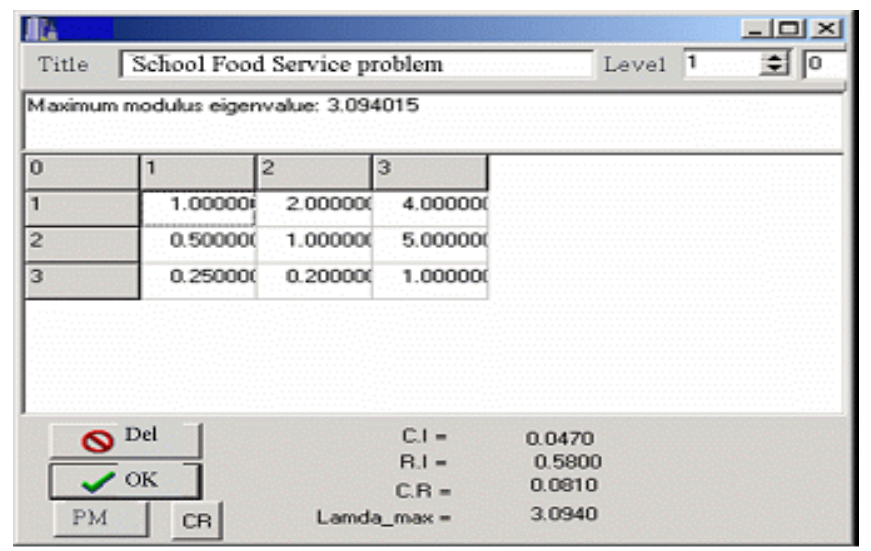

Figure 5. Sample output of pair-wise comparison

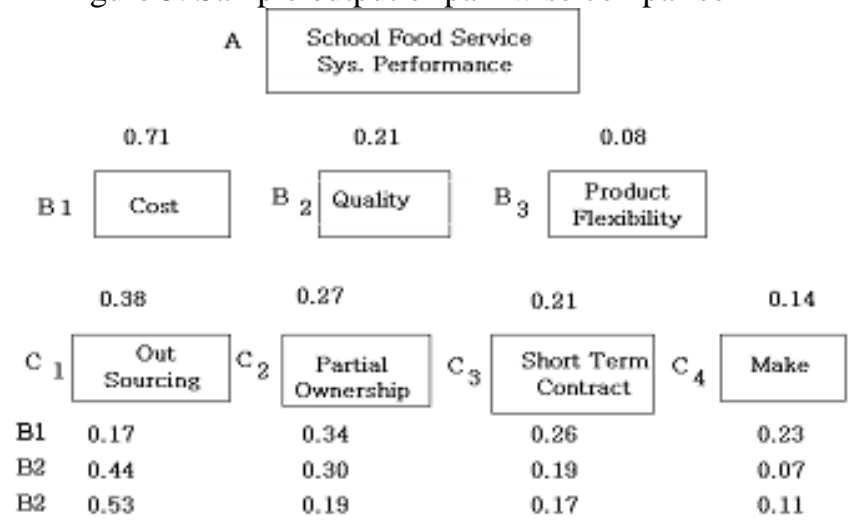

Figure 6. Sample output of evaluation by level

Figure 6 shows a sample output of alternative generation and construct the decision structure of the sample result of an evaluator. The final result of restaurant by fuzzy-AHP is given by:

$\mathrm{C} 1(0.38)>\mathrm{C} 2(0.27)>\mathrm{C} 3(0.21)>\mathrm{C} 4(0.14)$.

Table 2. Sample output of pair-wise matrix

\begin{tabular}{|l|llll|l}
\hline A & B1 & B2 & B3 & Eigen Val. & \\
B1 & 1.00 & 2.00 & 4.00 & 0.71 & $\lambda_{\text {max }}=3.09$ \\
B2 & 0.50 & 1.00 & 5.00 & 0.21 & $\mathrm{Cl}=0.0815$ \\
B3 & 0.25 & 0.20 & 1.00 & 0.08 & CR $=0.14$ \\
\hline
\end{tabular}

\begin{tabular}{|c|ccccc|l}
\hline B1 & C1 & C2 & C3 & C4 & Eigen Val & \\
\hline C1 & 1.00 & 0.33 & 0.50 & 0.50 & 0.17 \\
C2 & 3.00 & 1.00 & 1.00 & 2.00 & 0.34 \\
C3 & 2.00 & 1.00 & 1.00 & 1.00 & 0.26 & $\lambda_{\operatorname{lnx}}=5.760$ \\
C4 & 2.00 & 0.50 & 1.00 & 1.00 & 0.23 & $\mathrm{Cl}=0.190$ \\
$\mathrm{CR}=0.170$
\end{tabular}

\begin{tabular}{|c|ccccc|c|}
\hline B2 & C1 & C2 & C3 & C4 & Eigen Val & \\
\hline C1 & 1.00 & 2.00 & 4.00 & 5.00 & 0.44 \\
C2 & 0.50 & 1.00 & 3.00 & 7.00 & 0.30 \\
C3 & 0.25 & 0.33 & 1.00 & 5.00 & 0.19 & \\
C4 & 0.20 & 0.14 & 0.20 & 1.00 & 0.07 & Cl $=0.0275$ \\
CR $=0.024$ \\
\cline { 5 - 5 }
\end{tabular}

\begin{tabular}{|c|ccccc|l}
\hline B3 & C1 & C2 & C3 & C4 & Eigen Val & \\
\hline C1 & 1.00 & 3.00 & 9.00 & 4.00 & 0.53 & \\
C2 & 0.33 & 1.00 & 1.00 & 1.00 & 0.19 \\
C3 & 0.11 & 1.00 & 1.00 & 3.00 & 0.17 & $\lambda_{\max }=5.760$ \\
C4 & 0.25 & 1.00 & 0.33 & 1.00 & 0.11 & $\mathrm{Cl}=0.190$ \\
$\mathrm{CR}=0.170$
\end{tabular}


3) Integration of Individual Evaluations: Step 3

In this step, we integrated the results of the reviewers by the majority rule. The individual results of 4 reviewers are given by:

Reviewer 1: C1 > C3 > C2 > C4, Reviewer 3: C2 > C1 > C3 > C4,

Reviewer 2: C2 > C1 > C3 > C4, Reviewer 4: C1 > C2 > C4 > C3

Using the Heuristic 1, Heuristic 2 and Fuzzy Set Ranking method, we integrated as shown in Table 3:

Table 3. Results of Integrated Priority

\begin{tabular}{|l|l|}
\hline Majority Rule used & Priority by alternative \\
\hline 1. Heuristic method & C2 $>$ C1 $>$ C $3>$ C4 \\
\hline 2. Fuzzy set ranking method & $\mathrm{C} 1>$ C2 $>$ C $3>$ C4 \\
\hline
\end{tabular}

In this example, the best restaurant alternative for food supply planning is known to be alternative C1 (outsourcing)

\section{Optimizing Restaurant Locations and Service Level}

According to the decision of restaurant type, the design activity of restaurant facility planning improves customer centric services and the restaurant performance by implementing optimizing method (Hwang, 2000, 2003). We consider a restaurant chain system which is consisted of several central restaurants and its general restaurants.

\subsection{Optimal Location and Number of Facilities Using Stochastic Set-Covering Problem}

The objective of this section is to determine the minimum number of restaurant facilities among a discrete set of possible location sites so that the probability of each general restaurants to be covered is not less than a critical value (required service level) (Ally and White,1998). We formulate this problem using a stochastic set-covering problem which can be solved by $0-1$ programming method. Also we develop a computer program for this problem and applied to a sample problem. The results of sample problems for restaurant facility well show the impact of critical values and the number of possible location sites. Following assumptions are used in this study: 1) a restaurant planning is considered, 2) required service level is considered as performance measure of system, 3) covering probability is considered, and 4) GISdistance measure is assumed (Hwang, 2000).

To formulate the problem, we define the system cost $F_{i j}$ as the sum of transportation cost of demand per period. To compute the amount of system cost to transport from $S_{o}$ to $R_{j}$, we define following notations:

$r_{i}\left(X R_{i}, Y R_{i}\right)$ : Location of students,

$S_{j}\left(X S_{j}, Y S_{j}\right)$ : Possible location of a public facility

$F_{i j}\left(R_{i}, S_{j}\right)$ : Travel cost incurred between $r_{i}$ and $S_{j}$,

$A_{i}$ : required service level, $\mathrm{m}$ : Number of students

$\mathrm{n}:$ number of public system locations

$C_{j} \quad$ : cost of location a public service at site $\mathrm{j}$

$X_{j}\left[\begin{array}{cc}1 & \text { if a public service facility is located at site } j \\ 0 & \text { otherwise }\end{array}\right.$

$r_{i}$ : minimum allowable probability that student $I$

$F_{i j}=c_{i} \cdot \operatorname{Dist}\left(S_{j}, R_{i}\right) \cdot D_{i} \cdot \operatorname{Exp}\left(\alpha_{i} \cdot \operatorname{Tim}\left(S_{j}, R_{i}\right)\right)$

where, distance $\operatorname{Dist}\left(S_{j}, R_{i}\right)$ is given by lp-distance

$$
\operatorname{Dist}\left(S_{j}, R_{i}\right)=\left(\left|X S_{j}-X R_{i}\right|^{p}+\left|Y S_{j}-Y R_{i}\right|^{p}\right)^{1 / p}
$$

Using well known the deterministic set-covering problem, we formulate a probabilistic set covering problems under the assumptions : 1) restaurant facilities are always in available state, and 2) the 
traveling time is considered as a random variable with known distribution. When we consider the required service level as a random variable state, a probabilistic formulation P1 can be given by:

$$
\begin{aligned}
& \text { P 1: Minimize } \sum_{j=1}^{n} C_{j} X_{j} \text {, } \\
& \text { Subject to } \sum_{j=1}^{n} a_{i j} \cdot X_{j} \geq 1, \quad \text { for all } i=1, \cdots, m \\
& X_{j}=(0,1), \quad \text { for all } j=1, \cdots, n \\
& \text { where, } a_{i j}=\left[\begin{array}{lll}
1, & \text { if } & \operatorname{Pr} o b\left(F_{i j} \leq A_{i}\right) \geq r_{i} \\
0, & \text { if } & \text { otherwise }
\end{array}\right.
\end{aligned}
$$

This problem can be solved by a 0-1 programming algorithm. The covering coefficient $a_{i j}$ in P1 is determined under the assumption that a public service facility is always in available state. This assumption can be relaxed by considering the probability of a public service facility being in available state.

Let $b_{j}=$ Prob. (a public service facility is in available state), and $P_{i j}=\operatorname{Prob.~}\left(R_{i}\right.$ is covered by $S_{j}$ ), then, $P_{i j}=a_{i j} b_{j}$, and $q_{i j}=1-P_{i j}$,

Thus, Prob. [ $R_{i}$ is covered by some of the available supply center ] $=1-\prod_{j=\theta(x)} q_{i j}$

where, $\theta(X)=\left(j \mid X_{j}=1, \quad j=1, \cdots, n\right)$.

The equation (3) leads us to develop another formulation P2 as ;

$$
\begin{aligned}
& \text { P 2: Minimize } \sum_{j=1}^{n} X_{j} \text {, } \\
& \text { Subject to } 1-\prod_{j=\theta(x)} q_{i j} \geq l_{i} \text {, for } i=1, \cdots, m \\
& X_{j}=(0,1) \text {, for all } j=1, \cdots, n
\end{aligned}
$$

where, $l_{i}$ is the probability that $R_{i}$ is covered by some available supply centers with its maximum value to $\left(1-\prod q_{i j}\right)$. To make the solution procedure of P2 more effective, the first constraint can be transformed as follows:

$$
1-\prod_{j=\theta(x)} q_{i j}=1-\prod_{j=1}^{n}\left(q_{i j}\right) X_{j} \geq l_{i} \text {, for } R_{i} \quad i=1, \cdots, m
$$

thus, $\quad 1-\prod_{j=1}^{n}\left(q_{i j}\right) X_{j} \geq 1-l_{i}$

Taking a logarithmic transformation, equation (4) becomes

$$
-\sum_{j=1}^{n}\left(\log q_{i j}\right) X_{j} \geq-\log \left(1-l_{i}\right),
$$

Let $s_{i j}=-\log q_{i j}$, and $W_{i}=-\log \left(1-l_{i}\right)$, then, P2 can be reformulated as;

$$
\begin{aligned}
& \text { P } 3 \text { : Minimize } \sum_{j=1}^{n} X_{j} \text {, } \\
& \text { Subject to } \sum_{j=1}^{n} s_{i j} X_{j} \geq W_{i} \text {, for } i=1, \cdots, m
\end{aligned}
$$




$$
X_{j}=(0,1), \text { for all } j=1, \cdots, n
$$

This is the form of standard $0-1$ programming and can be solved by an appropriate algorithm. We developed GUI-type program for this stochastic set-covering problem and demonstrated sample results in following section. We developed the window program to solve this problem.

\subsection{Sample Problem of Public Service Facility Location}

The objective of this problem is the optimal location of central restaurants facilities to be open and the minimum number of central restaurants to cover the general restaurant's service requirements. We used the stochastic set covering problem based on GIS.

The major input data are:

- Number of general restaurant: 31,

- Number of possible locations of central supply center: 5 ,

- Covering probability: 0.80 (it can be changed),

- All the locations of general restaurant and of possible location of central restaurant are given by characteristics address and these are automatically converted into visual points in the GIS screen,

- Availability of possible school: $1^{\text {st }}=0.96,2^{\text {nd }}=0.97,3^{\text {rd }}=0.98,4^{\text {th }}=0.97,5^{\text {th }}=0.99$,

- Covering probability: 10\% $50 \%$,

Visual locations of general restaurant and central restaurant based on GIS window are shown in Figure 7. The results of stochastic set covering program outputs are shown in Table 4. We can say the possible locations which guarantee the student's walking distance within $1.1 \mathrm{~km}$ and with the service probability $50 \%$. The number of alternative of optimal location set is given by Figure 7 .

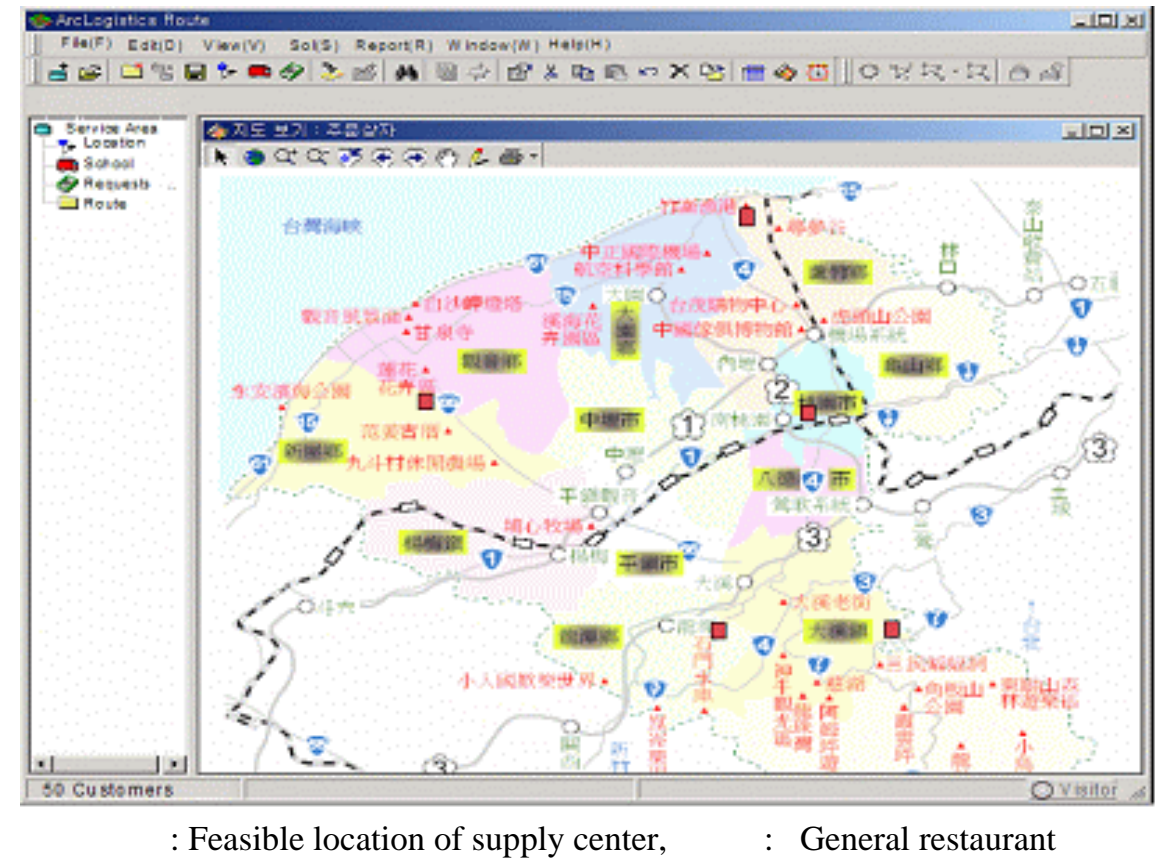

Figure 7. Mapping the location Data into GIS window and results of sector clustering.

Table 4. Sample output of sector clustering

Input Data

Cover matrix when cover probability $=80 \%$

Number of general restaurant: 31

Number of feasible location (a, b, c, d, e) $=5$

Number of available supply center $=5$ 


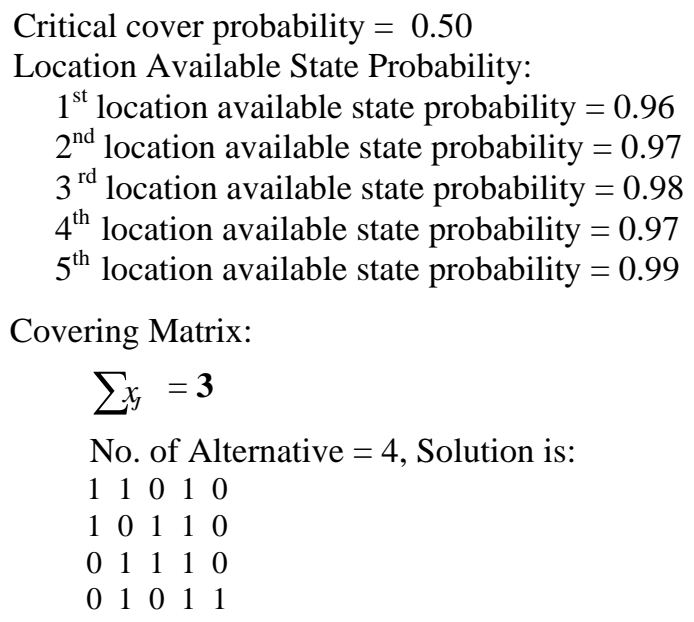

We can conclude that to meet the customer request three supply center needed and with 4 combinations as; (a, b, d), (a, c, d), (b, c, d), and (b, d, e). We can show the covering area of this supply center and also each of these travel routing schedules in the GIS window.

\section{Conclusion}

In this paper, a three-step model is proposed for the restaurant planning problem. In step 1, we developed an intranet/internet based three-step solution builder to evaluate the alternatives for restaurant type using brainstorming and AHP. In step 2, we developed a stochastic set covering problem to solve the optimal requirement and locations of supply centers for general restaurant to cover all the customers within a critical service level. In the last step, we assigned the general restaurants to one of possible supply centers which can provide within a certain required service level. Finally we developed a GUI-type computer program for the proposed method for restaurant planning using GIS and Geo-Database. We applied the proposed model to a restaurant planning problem in Taoyuan area of Taiwan. The computational results showed that the proposed method is very effective on a set of test problems. For the academic users, we would provide this software and user manual for educational purposes.

\section{References}

Aly A. and White J.A. (1998), Probabilistic Formulation of Emergency Service Facility Location Problem, Journal of Operations Research Society, 29, 1167-1179.

Apple, J. M. and Rickleres, H. V., (1987), “Material Handling and Storage, production Handbook ”, Jon Wiley, Newyork.

Barbarosoglu, G. \& Yazgas, T. (1997), Application of the analytic hierarchy process to the supplier selection problem, Production and Inventory Management Journal, Vol.38, No.1, pp.14-21.

Brown, D. M., \& Hoover, L. W. (1990). Productivity measurement in foodservice: Past accomplishments - a future alternative. Journal of the American Dietetic Association, 90, 973-978.

Clark, J. R. (1997). Improving catering productivity. Cornell Hotel and Restaurant Administration Quarterly, 38(4), 60-67.

ESRI Inc. (1998), "ArcView Network Analyst", An ESRI White Paper.

Francis, R. L. and White, J. A. (1974), “Facility Layout and Location, An Analytical Approach”, Prentice-Hall, Englewood Cliffs, N. J.

Gendrearu, M., A. Hertz and G. Raport (1991), "A Tabu Search Heuristics for Vehicle Routing Problem", CRT-77, Centre de Recherche, University of Montreal, Management Science. 
Heung Suk Hwang, (2000), Integrated GA-VRP Solver for Multi-Depot System, Int. Jnl. of Computers and Industrial Engineering 27 ${ }^{\text {th }}$ Conference Proceeding, pp. 1-6.

Heung Suk Hwang, (2003), Integrated Logistics/SCM System, Jnl. of the Korean Society of Supply Chain Management, Vol. 3, No. 2.

Heung Suk Hwang, (2003), Customer Responsive Pickup and Delivery System Based on GIS, Int. Jnl. of Agile Manufacturing, Vol. 6, No.2.

Heung Suk Hwang, (2003), A Pickup and Delivery Problem Based on AVL and GIS, Int. Jnl. of Industrial Engineering Management Systems, V0l. 2, No. 1.

Lieux, E. M., \& Winkler, L. L. (1989). Assessing productivity of foodservice systems in nutrition programs for the elderly. Journal of the American Dietetic Association, 89, 826-829.

Manning, C. K., \& Lieux, E. M. (1991). Comparison of labor productivity in nutrition program for the elderly. Journal of Foodservice Systems, 6, 155-166.

Manning, C. K., \& Lieux, E. M. (1995). Labor productivity in nutrition programs for the elderly that use a commissary-satellite production system. Journal of Foodservice Systems, 8, 187-200.

Mayo, C. R., \& Olsen, M. D. (1984). Variables that affect productivity in school foodservices. Journal of the American Dietetic Association, 84, 187-193.

Potvnt Y. (1996), The Vehicle Routing Problem with Time Window Part 1 Tabu Search, Informs Journal on Computing. Vol. 8, No. 2, pp. 158-164.

Saaty, T. L. ( 1981), The Analytic Hierarchy process, McGraw-Hill.

Shannon, R.E,(1986), The Amalgamation of Group Opinions or Evaluations, Journal of Industrial Engineering, Vol. 19, No.6.

Tomkins, J. A., White, J. A., et al. ((1996), “Facility Planning”, Second Edition, John Wiley and Sons.

Zahedi, F. (1986), The analytic Hierarchy Process - A Survey of the Method and it's Applications, Interfaces, Vol. 16, No. 4, pp. 96-104. 